\title{
A rediscussion on the eclipsing binary YY Canis Minoris
}

\author{
P. Vivekananda Rao, M.B.K. Sarma, and K.D. Abhyankar \\ Centre for Advanced Study in Astronomy, Osmania University, Hyderabad 500 007, India
}

Received November 19, 1998; accepted April 29, 1999

\begin{abstract}
We have reanalysed the $U B V$ light curves of Abhyankar (1962b) using the 1993 version of WilsonDevinney computer programme with a view to derive a consistent solution in all the three passbands and to answer the discordant opinions on the general picture of YY CMi. Initially, a preliminary unspotted solution was obtained and a photometric mass ratio was derived. From the present analysis, we obtained a mass ratio of $q=0.89$ which differs from the mass ratio of 0.8 derived by Giuricin \& Mardirossian (1981) and 0.65 derived by Abhyankar (1962b) but agrees with the value of 0.885 derived by Niarchos et al. (1998). Finally, the light curves were modelled by introducing a spot on the cooler secondary component to represent the observed light curve asymmetries. Assuming the mass of the primary component to be $1.56 m_{\odot}(\mathrm{F} 1 \mathrm{~V})$, the absolute elements of YY CMi are found to be $m_{\mathrm{c}}=1.39 m_{\odot}, R_{\mathrm{h}}=2.52 R_{\odot}, R_{\mathrm{c}}=2.38 R_{\odot}$, $\log L_{\mathrm{h}}=1.13 L_{\odot}, \log L_{\mathrm{c}}=0.86 L_{\odot}, \log g_{\mathrm{h}}=3.83$ and $\log g_{\mathrm{c}}=3.83$. The primary and secondary components are found to be slightly overluminous and bigger in size when compared to stars of the same mass. In the H-R diagram $\left(\log T_{\mathrm{e}}\right.$ versus $\left.\log L, \mathrm{ZAMS}\right)$, both the components are above but near the ZAMS suggesting that both of them have left the main sequence and have come into contact. Our results essentially agree with those of Niarchos et al. (1998) based on $V$ passband only.
\end{abstract}

Key words: stars: YY CMi — stars: binaries: eclipsing stars: fundamental parameters

\section{Introduction}

The light variability of the eclipsing binary YY CMi (HD 67100) was discovered by Morgenroth (1934). It is classified as a Beta Lyrae type eclipsing binary in the variable star catalogue of Kukarkin et al. (1969) who gave

Send offprint requests to: P. Vivekananda Rao,

e-mail: pvr@ouastr.ernet.in a period of 1.0940253 day and a spectral type of F5. Complete photoelectric light curves in $U B V$ passbands were obtained by Abhyankar (1962a,b) who gave a period of 1.0940197 determined on the basis of observed minima times spread over a period of 24 yrs. Applying Russel \& Merrill's method (1952) he found the primary eclipse to be a transit and concluded that it is a detached binary composed of an F8III primary and an A5V secondary. Giuricin \& Mardirossian (1981) have reanalysed Abhyankar's (1962a,b) three-colour photoelectric observations using Wood's (1972) model and found that their photometric elements differed appreciably from those of Abhyankar's, leading to a contact configuration. They have expressed the view that Wood's model (which treats the stars as triaxial ellipsoids) used by them for the analysis may not handle the contact systems very well and hence the derived elements may be questionable. In view of the existing discordant opinions on the general picture of YY CMi, we felt it worthwhile to reanalyse the three light curves of Abhyankar (1962a) using the synthetic light curve analysis method of Wilson \& Devinney (1971) according to their improved version of 1993. In the following, we give details of our analysis and its results.

In the meantime Niarchos et al. (1998) have obtained new photometric observations of YY CMi in $V$ passband and analysed them by the Wilson-Devinney method. Both theirs and our results confirm the contact nature of the binary and allow us to compare the solutions obtained from the two sets of observations separated by a span of 30 years.

To our knowledge no radial velocity studies of YY CMi are yet available in the literature.

\section{Photometric solutions}

Although YY CMi exhibits well defined eclipses, the analysis of its light curves is quite difficult because:

a) the light curves show significant anomalies, and

b) the spectroscopic mass-ratio is not known. 
The asymmetries in the light curves are pronounced around the two maxima. The O'Connell effect amounts to $\Delta m=$ Max I - Max II $=0.025,0.030$ and 0.025 in $U$, $B$ and $V$ bands, respectively. Light curve asymmetries can be explained by sunspot-like dark spots on the components (Binnendijk 1960; Hilditch 1981; Linnell 1982). Mullan (1975) and several investigators felt the need to place spots on contact binaries to obtain better light curve solutions (e.g. Van Hamme \& Wilson 1985; Milone et al. 1987; Niarchos et al. 1992, 1994, 1998). Moreover, strong magnetic activity on late-type stars, and the existence of large spots on the surfaces of these stars are suggested by the high angular momentum loss in very close and contact binaries (van't Veer \& Maceroni 1988, 1989; Maceroni et al. 1990). Hence, the light curve anomalies in YY CMi can be tackled by invoking cool and/or hot spots on the components since YY CMi exhibits light curve anomalies in all the three bands. The lack of a spectroscopic mass ratio does not affect the photometric solution, although it makes it more difficult. The photometric solution was carried out by us in the following way.

\subsection{Unspotted solution}

First, we tried to solve the $U, B$ and $V$ light curves (individual points) of Abhyankar (1962a) without any spots on the two components using the Wilson \& Devinney (W-D) (1971) method according to their improved version of 1993. For initiating the W-D programme, we used the values of a few of the required parameters given by Giuiricin \& Mardirossian (1981), as initial parameters. We treated the following parameters as fixed: the temparature, $T_{\mathrm{e}, \mathrm{h}}(6400 \mathrm{~K})$ of the hot component (cf. $6360 \mathrm{~K}$ used by Niarchos et al. (1998) by assuming a spectral type of F6); the mass-ratio, $q(0.8)$ of the system; the ratio of the surface rotation rates to synchronous rotation rates, $F_{\mathrm{h}}$ and $F_{\mathrm{c}}$ as unity; the limb darkening coefficients, $x_{\mathrm{h}}$ and $x_{\mathrm{c}}$; the albedos, $A_{\mathrm{h}}$ and $A_{\mathrm{c}}$; and the gravity darkening coefficients, $g_{\mathrm{h}}$ and $g_{\mathrm{c}}$ of the hotter and cooler components, respectively. According to the principles of the W-D method (Milone et al. 1995), we adjusted the following parameters; the inclination, $i$ : the temperature of the cool component, $T_{\mathrm{e}, \mathrm{c}}$; the surface potential of the hotter star $\Omega_{\mathrm{h}}$; the relative monochromatic luminosity of the hot component, $L_{\mathrm{h}}$ and the third light $l_{3}$. Sufficient number of runs of the DC programme was made until the sum of the residuals, $\Sigma W(\mathrm{O}-\mathrm{C})^{2}$ showed a minimum and the corrections to the parameters became smaller than their probable errors. In order to check the internal consistency of the results (Popper 1984), separate solutions, for each of the $U, B$ and $V$ light curves, were made. The study of Abhyankar (1962b) suggested YY CMi to be a semidetached system while Giuricin \& Mardirossian (1981) and Niarchos et al. (1998) indicated it to be a contact binary. Hence, to ascertain the nature of the system, we analysed the light curves using W-D method with two different modes viz; mode-4 (semi-detached with primary filling the Roche lobe) and mode-3 (contact). The analysis with mode-4 (semi-detached with primary filling the Roche lobe) did not yield convergency, while the analysis with mode-3 (contact) yielded converged solutions and hence we proceeded with the contact nature of the system only. The results of the analysis, with mode- 3 , of individual $U, B$ and $V$ light curves indicated that the individual solutions are consistent and that a combined solution for $U, B$ and $V$ is adequate to derive the system parameters. Further, the fit of these results to the observations were found to be good except for the reduced light in the phase interval from 0.6 to 0.93 .

Using the derived luminosities, the magnitude and colour of the comparison star $[V=8.68, B=8.73$ and $U=8.84]$ and the corresponding differential magnitudes for unit luminosity at the quadrature $[V=+0.320$, $B=-0.005$ and $U=-0.130]$, we derived the colours $(B-V)$ and $(U-B)$ of the primary, hot component to be 0.32 and -0.06 .

Assuming no space reddening, the derived colours for the primary component corresponds to an average temperature of $7000 \pm 100 \mathrm{~K}$ (Allen 1976; Popper 1980; SchmidtKaler 1982). Hence, a reanalysis of the individual light curves as well as a combined solutions for $U, B$ and $V$ colours was made with $T_{\mathrm{e}, \mathrm{h}}=7000 \mathrm{~K}$ and various values of $q(0.7$ to 1.0$)$ as fixed parameters. In this analysis the discordant points in the phase range 0.6 to 0.93 were removed from consideration. From a plot of the resulting $\Sigma W(\mathrm{O}-\mathrm{C})^{2}$ against $q$ it was found that the minimum occurs at $q=0.90$. The individual solution with $q=0.90$ are given in Cols. 2, 3 and 4 of Table 1.

Taking the average parameters of these solutions as preliminary elements a final combined solution was obtained keeping $i, T_{\mathrm{h}}, \Omega_{\mathrm{h}}$ and $L_{\mathrm{h}}$ as adjustable parameters and $x_{\mathrm{h}}, x_{\mathrm{c}}, g_{\mathrm{h}}, g_{\mathrm{c}}, A_{\mathrm{h}}$ and $A_{\mathrm{c}}$ as fixed parameters. It is given in Col. 5 of Table 1 . One can notice that $l_{3}$ is absent in all the three colours in the individual as well as in the combined solutions. The theoretical curves obtained from the elements given in Table 1, Col. 5 are shown in Figs. $1 \mathrm{a}$ and $1 \mathrm{~b}$ as solid lines. In these figures, the open diamonds $(V)$, open triangles $(B)$ and open squares $(U)$ represent the individual observations of Abhyankar (1962a).

\subsection{Spotted solution}

Figures $1 \mathrm{a}$ and $1 \mathrm{~b}$ do show that the theoretical light curves of the unspotted solutions fit the data quite well except the reduced light in the phase range 0.6 to 0.93 . So we tried to obtain a better fit by introducing a cool spot on the surface of the secondary (cooler) component. The spot region was centered in the co-latitude ranges 90 to 60 degrees, while the other spot parameters namely longitude (measured counter-clockwise on the secondary as 
Table 1. YY CMi: Elements obtained from the solution of individual and combined $V B U$ light curves using W-D method

\begin{tabular}{|c|c|c|c|c|c|}
\hline Element & $V$ & $B$ & $U$ & $\begin{array}{c}\text { Combined } V B U \\
\text { unspotted solution }\end{array}$ & $\begin{array}{l}\text { Combined } V B U \\
\text { spotted solution }\end{array}$ \\
\hline 1 & 2 & 3 & 4 & 5 & 6 \\
\hline$T_{\mathrm{e}, \mathrm{h}} K$ & $7000^{*}$ & $7000^{*}$ & $7000^{*}$ & $7000^{*}$ & $7000^{*}$ \\
\hline$T_{\mathrm{e}, \mathrm{c}} K$ & $6152 \pm 29$ & $6127 \pm 31$ & $6149 \pm 34$ & $6140 \pm 44$ & $6161 \pm 36$ \\
\hline$q$ & $0.90 \pm 0.02$ & $0.90 \pm 0.02$ & $0.90 \pm 0.02$ & $0.90 \pm 0.02$ & $0.89 \pm 0.02$ \\
\hline$i^{0}$ & $78.83 \pm 0.04$ & $78.87 \pm 0.04$ & $78.68 \pm 0.04$ & $78.67 \pm 0.05$ & $78.76 \pm 0.04$ \\
\hline$\Omega_{\mathrm{h}}=\Omega_{\mathrm{c}}$ & $3.5541 \pm 0.0121$ & $3.5511 \pm 0.0119$ & $3.5614 \pm 0.0126$ & $3.5578 \pm 0.0153$ & $3.5521 \pm 0.0148$ \\
\hline \%overcontact & $6 \%$ & $7 \%$ & $5 \%$ & $5 \%$ & $3 \%$ \\
\hline$r_{\mathrm{h}}$ pole & $0.3690 \pm 0.0011$ & $0.3703 \pm 0.0011$ & $0.3684 \pm 0.0011$ & $0.3698 \pm 0.0025$ & $0.3691 \pm 0.0013$ \\
\hline side & $0.3889 \pm 0.0011$ & $0.3901 \pm 0.0011$ & $0.3882 \pm 0.0011$ & $0.3897 \pm 0.0026$ & $0.3890 \pm 0.0014$ \\
\hline back & $0.4214 \pm 0.0012$ & $0.4226 \pm 0.0012$ & $0.4207 \pm 0.0012$ & $0.4222 \pm 0.0025$ & $0.4203 \pm 0.0015$ \\
\hline$r_{\mathrm{c}}$ pole & $0.3515 \pm 0.0017$ & $0.3528 \pm 0.0017$ & $0.3509 \pm 0.0017$ & $0.3523 \pm 0.0030$ & $0.3501 \pm 0.0020$ \\
\hline side & $0.3694 \pm 0.0017$ & $0.3706 \pm 0.0017$ & $0.3687 \pm 0.0017$ & $0.3702 \pm 0.0029$ & $0.3664 \pm 0.0019$ \\
\hline back & $0.4008 \pm 0.0018$ & $0.4020 \pm 0.0018$ & $0.4011 \pm 0.0018$ & $\begin{array}{c}0.4019 \pm 0.0030 \\
V 0.6343 \pm 0.0013\end{array}$ & $\begin{array}{c}0.3978 \pm 0.0020 \\
V 0.6407 \pm 0.0007\end{array}$ \\
\hline$L_{\mathrm{h}}$ & $0.6366 \pm 0.0010$ & $0.6626 \pm 0.0010$ & $0.6955 \pm 0.0018$ & $\begin{array}{c}B 0.6626 \pm 0.0014 \\
U 0.6934 \pm 0.0020 \\
V 0.3657\end{array}$ & $\begin{array}{c}B 0.6690 \pm 0.0008 \\
U 0.6998 \pm 0.0012 \\
V 0.3593\end{array}$ \\
\hline$L_{\mathrm{c}}$ & 0.3634 & 0.3374 & 0.3405 & $\begin{array}{l}B 0.3374 \\
U 0.3066\end{array}$ & $\begin{array}{c}B 0.3310 \\
U 0.3002\end{array}$ \\
\hline${ }^{+} l_{3}$ & 0.0 & 0.0 & 0.0 & $\begin{array}{ll}V & 0.0 \\
B & 0.0 \\
U & 0.0\end{array}$ & $\begin{array}{ll}V & 0.0 \\
B & 0.0 \\
U & 0.0\end{array}$ \\
\hline$x_{\mathrm{h}}=x_{\mathrm{c}}$ & $0.51^{*}$ & $0.64^{*}$ & $0.83^{*}$ & 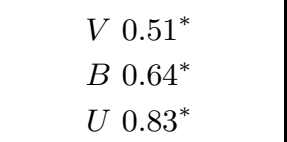 & 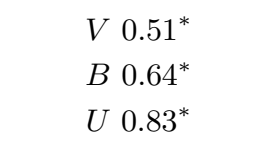 \\
\hline$A_{\mathrm{h}}=A_{\mathrm{c}}$ & $0.5^{*}$ & $0.5^{*}$ & $0.5^{*}$ & $0.5^{*}$ & $0.5^{*}$ \\
\hline$g_{\mathrm{h}}=g_{\mathrm{c}}$ & $0.32^{*}$ & $0.32^{*}$ & $0.32^{*}$ & $0.32^{*}$ & $0.32^{*}$ \\
\hline$\Sigma(\text { res })^{2}$ & 0.0015 & 0.0018 & 0.0025 & 0.0404 & 0.0173 \\
\hline
\end{tabular}

* Fixed parameters.

+ In units of total system light at phase 0.25 .

Table 2. YY CMi: Comparison of the spot parameters obtained from the present studies and by Niarchos et al. (1998) on the secondary component

\begin{tabular}{|c|c|c|c|}
\hline $\begin{array}{c}\text { Spot } \\
\text { Parameter }\end{array}$ & $\begin{array}{c}\text { Present } \\
\text { Studies }\end{array}$ & $\begin{array}{c}\text { Niarchos } \\
\text { et al. (1998) }\end{array}$ & $\begin{array}{c}\text { Niarchos } \\
\text { et al. (1998) }\end{array}$ \\
\hline & cool & cool & hot \\
\hline Co-latitude & $74.56^{\circ} \pm 2.81^{\circ}$ & $90^{\circ *}$ & $90^{\circ *}$ \\
Longitude & $89.68^{\circ} \pm 1.02^{\circ+}$ & $91.27^{\circ} \pm 0.91^{\circ+}$ & $201.26^{\circ} \pm 1.95^{\circ+}$ \\
Angular radius & $11.19^{\circ} \pm 0.96^{\circ}$ & $22.33^{\circ} \pm 4.51^{\circ}$ & $9.32^{\circ} \pm 2.69^{\circ}$ \\
T.F & $0.61 \pm 0.05$ & $0.84 \pm 0.09$ & $1.19 \pm 0.09$ \\
\hline
\end{tabular}

* Fixed parameter.

** T.F is the ratio of the local spot surface temperature to the local undisturbed surface temperature.

+ This is the longitude on the secondary measured counter-clockwise as defined in the W-D programme (see text).

The system is assumed to revolve clockwise. 


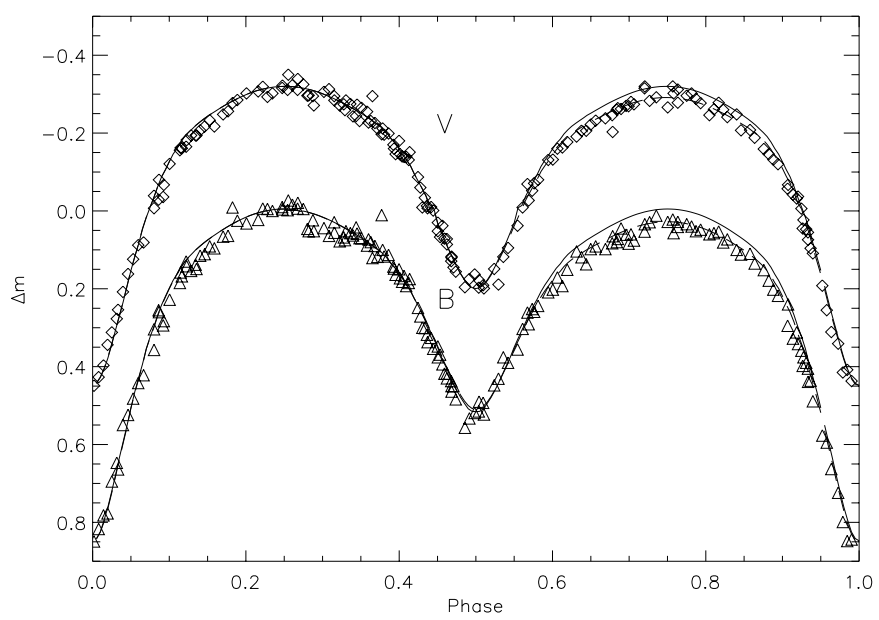

(a)

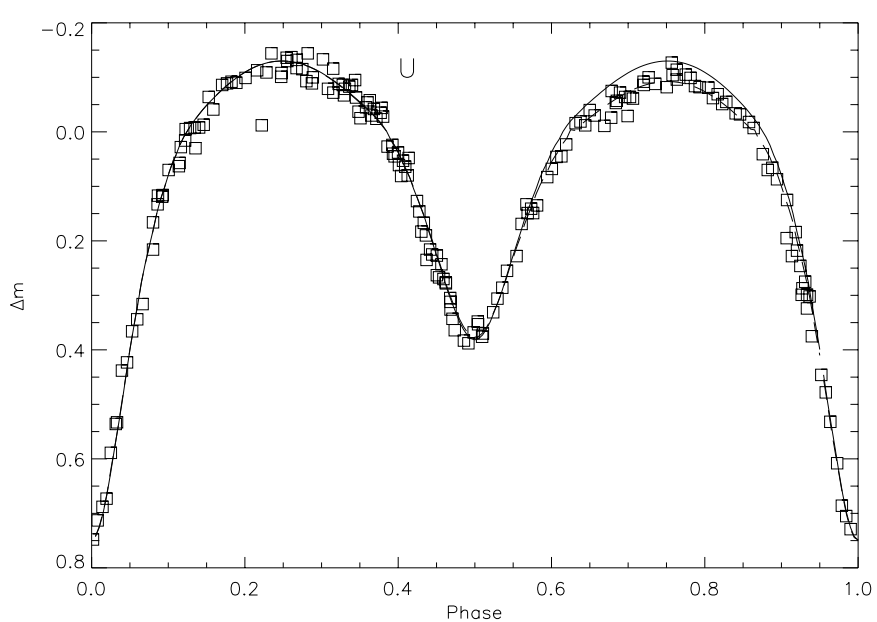

(b)

Fig. 1. a) YY CMi: Light curves in $V$ and $B$ passbands. Open diamonds represent $V$ observations, open triangles represent $B$ observations, solid lines represent unspotted solution and dashed lines represent spotted solution. b) YY CMi: Light curve for the $U$ passband. Open squares represent $U$ observations, solid line represents unspotted solution and dashed line represents spotted solution

defined in the Wilson-Devinney programme), angular size and temperature factor were first selected by trial and error to reproduce the light curve perturbations by using the parameters found in the unspotted solution in the LC (light curve) programme of the Wilson \& Devinney. The spot parameters were later subjected to a DC programme which gave the results listed in Table 2. These data were used for deriving the spotted solution of the light curve. The temperature of the hotter (primary) component was fixed at the value found in the unspotted solutions. The other parameters namely, $i, T_{\mathrm{c}}, \Omega_{\mathrm{h}}, q$ and $L_{\mathrm{h}}$ were varied. The differential corrections were calculated until the adjustments became smaller than their probable errors. The final results of the spotted solution is given in Table 1, Col. 6.

The theoretical light curves for the spotted solution are shown as dashed lines in Figs. 1a and 1b.

\subsection{Comparison with the solution of Niarchos et al. (1998)}

First we note that we have derived $T_{\mathrm{e}, \mathrm{h}}=7000 \mathrm{~K}$ as compared to their $T_{\mathrm{e}, \mathrm{h}}=6360 \mathrm{~K}$. But our value is preferred because it is based on $U B V$ colours while theirs is based on $V$ only. Similarly the fit of the spotted solution indicates that we do not need a hot spot on the secondary as found by Niarchos et al. (1998). The parameters, except for the longitude, of the cool spot are also different from theirs. This indicates a variation in the spot activity on the secondary star of the binary.

The difference in the longitude of the cool spot in the two solutions can be simply explained by the fact that the revolution of the binary is defined in a different way in the two solutions. In Niarchos et al. (1998) the system revolves counter-clockwise (according to Binary Maker 2.0), while in our solution the revolution is assumed to be clockwise. In both solutions the cool spot is better seen at phase 0.75, thus explaining the deficiency of light around Max II.

\section{Absolute elements}

Although spectroscopic observations are not available for this binary the $U B V$ photoelectric observations can be used for obtaining the tentative absolute elements. Using the derived luminosities (Table 1, Col. 6), the differential magnitudes, $\Delta m$, at the quadrature and the standard values of the magnitudes of the comparison star in the $U B V$ passbands (Sect. 2), we derived the following magnitudes and colours of the individual components: Hotter component $V=8.84, B-V=0.32, U-B=-0.06$, Cooler component $V=9.47, B-V=0.45, U-B=0.07$.

Assuming no interstellar reddening, the $(B-V)$ of the primary corresponds to a temperature of $7030 \pm 100 \mathrm{~K}$ which in turn corresponds to a spectral type of F1V (Allen 1976; Popper 1980; Schmidt-Kaler 1982). The above temperature agrees quite well with that of the fixed temperature $7000 \pm 100 \mathrm{~K}$ used for the primary component in the analysis. The $(B-V)$ of 0.45 for the secondary component corresponds to a spectral type of F5V $(6450 \mathrm{~K})$ and its derived temperature of $6145 \mathrm{~K}$ (Table 1, Col. 6) suggests a spectral type of F8V (Allen 1976; Popper 1980; SchmidtKaler 1982). This small discrepancy of about $300 \mathrm{~K}$ between the spectral types derived from the colour $(B-V)$ and effective temperature for the secondary component could be attributed to the uncertainties in the model atmospheres incorporated in the $\mathrm{W}$-D programme, appropriate for each spectral type. Keeping in view the bigger 
Table 3. YY CMi: Comparison of elements from various studies

\begin{tabular}{|c|c|c|c|}
\hline Parameter & $\begin{array}{c}\text { Giuricin } \\
\text { Mardirossian } \\
(1981)\end{array}$ & $\begin{array}{c}\text { Niarchos et al. } \\
\text { (1998) }\end{array}$ & $\begin{array}{l}\text { Present Studies } \\
\text { (spotted solution) }\end{array}$ \\
\hline Method & $\mathrm{a}$ & $\mathrm{b}$ & $\mathrm{b}$ \\
\hline$T_{\mathrm{e}, \mathrm{h}} K$ & $6400^{*}$ & $6360^{*}$ & $7000^{*}$ \\
\hline$T_{\mathrm{e}, \mathrm{c}} K$ & $5925 \pm 90$ & $5710 \pm 4$ & $6161 \pm 36$ \\
\hline$q$ & $0.8^{*}$ & $0.885 \pm 0.004$ & $0.89 \pm 0.02$ \\
\hline$i^{\circ}$ & $77.2 \pm 0.4$ & $79.47 \pm 0.07$ & $78.76 \pm 0.04$ \\
\hline$r_{\mathrm{h}}$ & $0.319 \pm 0.025(V)$ & $0.390 \pm 0.001^{\mathrm{a}}$ & $0.393 \pm 0.001^{\mathrm{a}}$ \\
\hline & $0.356 \pm 0.025(B)$ & & \\
\hline & $0.386 \pm 0.017(U)$ & & \\
\hline$r_{\mathrm{c}}$ & $0.386 \pm 0.043(V)$ & $0.368 \pm 0.002^{\mathrm{a}}$ & $0.371 \pm 0.002^{\mathrm{a}}$ \\
\hline & $0.404 \pm 0.053(B)$ & & \\
\hline & $0.386 \pm 0.019(U)$ & & \\
\hline $\log L_{\mathrm{h}} / L_{\odot}$ & $0.82 \pm 0.09$ & 0.91 & $1.13 \pm 0.01$ \\
\hline $\log L_{\mathrm{c}} / L_{\odot}$ & $0.71 \pm 0.08$ & 0.67 & $0.86 \pm 0.01$ \\
\hline$m_{\mathrm{h}}$ & $1.20^{*}$ & 1.25 & $1.56^{*}$ \\
\hline$m_{\mathrm{c}}$ & 0.96 & $1.12^{*}$ & 1.39 \\
\hline$R_{\mathrm{h}}$ & $2.1 \pm 0.2$ & 2.32 & $2.52 \pm 0.01$ \\
\hline$R_{\mathrm{c}}$ & $2.2 \pm 0.2$ & 2.20 & $2.38 \pm 0.01$ \\
\hline $\log g_{\mathrm{h}}$ & - & & $3.83 \pm 0.01$ \\
\hline $\log g_{\mathrm{c}}$ & - & & $3.83 \pm 0.01$ \\
\hline Sp.type(Pri) & F6 & F6 & F1V-IV \\
\hline$(\mathrm{Sec})$ & early G & & F5V-IV \\
\hline
\end{tabular}

sizes (radii) of both the components (see the following) we adopted a spectral type of F1V-IV for the primary and F5V-IV for the secondary component. Our improved solution based on $U B V$ photometry gives an earlier spectral type for the primary compared to other authors.

Since no spectroscopic studies exist for this system, neither the spectral types nor the individual masses of the components are presently available. Hence it is difficult to get reliable absolute elements for the components. However, to get tentative dimensions and masses of the components, we assumed the primary F1V component to obey the spectral type-mass relation of the normal main sequence stars (Andersen 1991). From this assumption one gets a mass of $1.56 m_{\odot}$ for the primary component. From the presently derived mass-ratio, $m_{\mathrm{c}} / m_{\mathrm{h}}=q=0.89$ and the assumed mass of $1.56 m_{\odot}$ for $m_{\mathrm{h}}$, one gets a mass of $1.39 m_{\odot}$ for the secondary component.

Using Kepler's 3rd law

$A^{3}=74.55\left(m_{\mathrm{h}}+m_{\mathrm{c}}\right) P^{2}$

and with a period of 1.0940197 (Abhyankar 1962b), one gets $A=6.41 R_{\odot}$ as the separation of the components. From the derived average fractional radii $[r=$ $\left.\left(r_{\text {pole }}+r_{\text {side }}+r_{\text {back }}\right) / 3\right]$ of 0.393 and 0.371 for the primary and secondary components respectively, one gets $A r_{\mathrm{h}}=R_{\mathrm{h}}=2.52 R_{\odot}$ and $A r_{\mathrm{c}}=R_{\mathrm{c}}=2.38 R_{\odot}$. The above derived radii of the primary and secondary components, $R_{\mathrm{h}}$ and $R_{\mathrm{c}}$, are larger than those of $2.07 R_{\odot}$ and $1.70 R_{\odot}$ of normal F1V and F5V stars respectively (Andersen 1991). This may be due to the evolution of both the primary and secondary components which are overfilling their Roche lobes $\left(r_{\mathrm{h}}^{*}=0.385, r_{\mathrm{c}}^{*}=0.363\right.$ for $q=0.89$; Plavec \& Kratochvil 1964) by $3 \%$. The system parameters are as given in Table 3 along with the elements and parameters given by Giuricin \& Mardirossian (1981) and Niarchos et al. (1998).

The bolometric corrections are from Popper (1980). According to Buser \& Kurucz (1978) a star with a $(B-V)$ colour of 0.32 and $\log g$ of 3.83 should have a temperature of about $7000 \mathrm{~K}$. This agrees quite well, within the errors of assumed parameters, with the temperatures of $7000 \pm 100 \mathrm{~K}$ used by us for the primary component in our analysis. 


\section{Conclusions}

A comparison of the presently derived parameters with those of Giuricin \& Mardirossian (1981) as well as Niarchos et al. (1998) indicate that YY CMi is an A type W UMa contact system.

From the present analysis, we obtained a mass ratio of $q=0.89$ which differs from the mass-ratio of 0.8 derived by Giuricin \& Mardirossian (1981) and 0.65 derived by Abhyankar but agrees with that found by Niarchos et al. (1998). This discrepancy in the values of the $q$ may be attributed to the different methods adopted in the analysis. The $U B V$ light curves of YY CMi show an O'Connell effect which suggests that there might be some source responsible for it. Like Niarchos et al. (1998) we attribute this effect to the spot activity on the surface of the cool secondary component. We have already noted the difference between the two spot models, which might indicate varying spot activity.

In order to understand the evolutionary status of both the components of YY CMi, we made a comparison of their positions with single stars in the $\log m$ versus $\log L$, $\log T$ and $\log R$ relations (ZAMS). The primary and secondary components are found to be slightly overluminous and bigger in size when compared to stars of the same mass. This evolution is much less than indicated by the solution of Niarchos et al. (1998). In the H-R diagram $\left(\log T_{\mathrm{e}}\right.$ versus $\log L$, ZAMS) both the components are above but near the ZAMS suggesting that both of them have left the main sequence and have come into contact.

Finally, we point out that our solution is based on photometric data only and hence the conclusions are tentative. It is suggested that spectroscopic observations would give a better understanding of the properties and the evolutionary status of this interesting contact binary.

Acknowledgements. We thank Shri. S. Muneer for his help in preparing the graphs on computer. We also thank the referee, Dr. P.G. Niarchos, for his comments which improved the quality of the paper. MBKS wishes to thank the Council of Scientific and Industrial Research (CSIR), New Delhi for financial assistance through Emeritus Scientists Scheme.

\section{References}

Abhyankar K.D., 1962a, Nizamiah Contrib., No. 1

Abhyankar K.D., 1962b, Z. fur Astrophys. 54, 25

Allen C.W., 1976, Astrophysical Quantities, 3rd edn. London: The Athlone Press

Andersen J., 1991, A\&AR 3, 91

Binnendijk L., 1960, AJ 65, 358

Buser R., Kurucz R.L., 1978, A\&A 70, 555

Giuricin G., Mardirossian F., 1981, A\&A 94, 391

Hilditch R.W., 1981, MNRAS 196, 305

Kukarkin N.E., Khopolov P.N., Efremov Yu.N., Kukarkina N.P., Kurochkin N.E., Medvedeve G.I., Perova N.B., Fedorovich V.P., Frolov M.S., 1969, General Catalogue of Variable Stars, Moscow

Linnell A.P., 1982, ApJS 50, 85

Maceroni C., Van Hamme W., van’t Veer F., 1990, A\&A 234, 177

Milone E.F., Wilson R.E., Hrivnak B.J., 1987, ApJ 313, 325

Milone E.F., Stagg C.R., Sugars B.A., et al., 1995, AJ 109, 359

Morgenroth O., 1934, Astr. Nachr. 252, 389

Mullan D.G., 1975, ApJ 198, 563

Niarchos P.G., Hoffmann M., Duerbeck H.W., 1992, A\&A 258, 323

Niarchos P.G., Hoffmann M., Duerbeck H.W., 1994, A\&AS 103, 39

Niarchos P.G., Mantegazza L., Poretti E., Manimanis V., 1998, A\&AS 133,7

Plavec M., Kratochvil P., 1964, Bull. Astron. Inst. Czechoslovakia 15, 165

Popper D.M., 1980, ARA\&A 18, 115

Popper D.M., 1984, AJ 89, 132

Russell H.N., Merrill J.E., 1952, Contr. Princeton Univ. Obs., No. 26

Schmidt-Kaler Th. 1982, in Landolt/Bornstein, Numerical data and functional relationship in Science and Technology, Scaifers K. \& Voigt H.H. (eds.). Springer-Verlag, Berlin, New Series, Group VI, Vol. 2(b), p. 453 and 31

Van Hamme W., Wilson R.E., 1985, A\&A 152, 25

van't Veer F., Maceroni C., 1988, A\&A 199, 183

van't Veer F., Maceroni C., 1989, A\&A 220, 128

Wood D.B., 1972, A Computer Program for Modelling NonSpherical Eclipsing Binary Systems Goddard Space Flight Center, Greenbelt, Maryland, U.S.A.

Wilson R.E., Devinney E.J., 1971, ApJ 166, 605 